\title{
Analysis of Cerebral Infarct Signal Intensity on Diffusion-Weighted MR Images Using Frequency Domain Techniques
}

\author{
S. S. Shanbhag ${ }^{1, *}$, G. R. Udupi ${ }^{2}$, K. M. Patil ${ }^{3}$, K. Ranganath ${ }^{4}$ \\ ${ }^{1}$ Electronics and Communication Engineering, Gogte Institute of Technology, Belgaum, 590001, India \\ ${ }^{2}$ Vishwanathrao Deshpande Rural Institute of Technology, Haliyal, 581329, India \\ ${ }^{3}$ Retired, Indian Institute of Technology (Madras), Belgaum, 590009, India \\ ${ }^{4}$ RAGAVS, Diagnostics and Research Center Pvt Ltd, Bangalore, 560011, India
}

\begin{abstract}
Early and accurate diagnosis of cerebral infarction plays a vital role in the implementation of successful treatment and thereby improving the quality of life. Diffusion Weighted Magnetic Resonance Imaging (DW-MRI) is a highly sensitive tool for the detection of early changes in the water diffusion that characterizes various brain pathologies, largely, cerebral infarctions. Studies were performed on a set of Diffusion Weighted (DW) images of the human brain (in the axial plane) to find the relationship between the light intensity High Frequency Power (HFP) values and the time course of cerebral infarction, and to present evidence in defining the stages of cerebral infarction. Analysis of the results show that the difference in the light intensity HFP values on the DW images for the subjects with cerebral infarction compared to their contralateral normal hemisphere, were highly significant $(\mathrm{p}<0.01)$ in the areas of the brain, where there was a high incidence of infarction. The relative increase in the light intensity HFP values (RHFP) for the subjects with cerebral infarction were in the range of $(153.06$ - 1780.43) times compared to their corresponding HFP values on the contralateral normal hemisphere. The observed RHFP values increased progressively with time and were at the peak for the examinations between 1 to 4 days and thereafter reduced to reach the minimum after 15 days. There was a negative correlation $(r=-0.74)$ observed between the RHFP values and the time stage of cerebral infarction. The evolution of the RHFP values observed subsequent to infarction is suggestive that they can be supportive in understanding the developmental stages of infarction and can be helpful in predicting the stage of infarction. The quantitative changes in the light intensity HFP values can be assessed to derive information about the early changes taking place in the brain tissue. Further their adoption in clinical diagnosis and treatment of cerebral infarction could be helpful and informative. In conclusion, the proposed method could positively assist the neuro-surgeons for speedy diagnosis and execution of treatment to protect the subjects from additional damage to their brain tissue.
\end{abstract}

Keywords Diffusion Weighted Imaging, High Frequency Power, Infarct, Magnetic Resonance Imaging, Signal Intensity

\section{Introduction}

Cerebral infarction is a leading cause of death and a major cause of long term disability. Post infarction treatments offered mainly depend on the timing after infarction to determine the stages of brain damage[1]. Recently, Diffusion Weighted Magnetic Resonance Imaging (DW-MRI), an imaging technique based on the sensitivity of magnetic reso nance to microscopic mobility of water molecules within tissues, has emerged to be applicable in the clinical diagnosis and treatment of cerebral infarction[2-4]. DW-MRI is considered to be the most sensitive tool for the diagnosis of

* Corresponding author:

supriya_sp@yahoo.com (S. S. Shanbhag)

Published online at http://journal.sapub.org/ajbe

Copyright (C) 2012 Scientific \& Academic Publishing. All Rights Reserved early cerebral infarction, mainly because of its exquisite sensitivity in the detection of early changes in the water diffusion that characterizes infarction[5]. While it takes hours to reach the diagnosis with conventional Magnetic Resonance Imaging (MRI), DW-MRI allows for the detection of cerebral infarcts within minutes after its onset and in addition provides clinically valuable information about the age of infarction[6-8]. Investigators from several previously published studies have described temporal evolution of the Apparent Diffusion Coefficient (ADC) values within lesions following cerebral infarction, to identify their usefulness in the clinical diagnosis of the progress of infarction and treatment. The ADC values reported from these studies have been computed from a single axial section (showing infarction) on the Diffusion Weighted (DW) images. Also the calculation of the $\mathrm{ADC}$ values considers only a small region 
within the infarcted area and further different studies propose diverse methods for calculating the ADC values[9-12]. Additionally, since the ADC values vary spatially within the infarcted area, a comprehensive understanding of the entire infarcted area would be necessary for better analysis[13-16].

In contrast to the available work concerning cerebral infarction using ADC values, the temporal evolution of infarct signal intensity on the DW images is less well documented in the literature $[1,17,18]$. Furthermore radiologists in a variety of clinical settings depend only on the DW images (signal intensity variations) to review subjects with cerebral infarctions. In this direction if the progression of the signal intensity on the DW images following cerebral infarction can be quantified and assessed, this information would surely aid the radiologists in early and accurate diagnosis of infarction. This would result in subjects receiving prompt treatment, which, in turn, improves their chances of survival and recovery. Therefore the objective of the present work is to determine the role of signal intensity changes on the DW images of the human brain in the axial plane in the early diagnosis and classification of the stages of cerebral infarction. Mainly an effort is made in grading the signal intensity changes on the DW images using the light intensity High Frequency Power (HFP) values.

The new method proposed in the present work takes care of the drawbacks listed earlier for the studies carried out in the past using $\mathrm{ADC}$ values for the diagnosis of cerebral infarction. In the proposed method all the axial sections showing cerebral infarction are taken into account and also the entire infarcted area on each axial section is considered to evaluate the light intensity HFP values. Therefore the method assures that there is no loss of information as it covers larger area of the brain and also expertise is not essential as the entire infarcted area is considered for carrying out the investigation. The light intensity HFP values obtained from the infarct subjects are compared to their corresponding HFP values from the contralateral normal hemisphere for the same subject to find the relative changes in the HFP values. The relative changes in the HFP values so obtained are employed to realize a quantitative model for the time course of the infarct signal intensity variations. The method could positively aid in the speedy and accurate radiological diagnosis of the infarct subjects and additionally provide important reference in judging the timing and developmental stages of cerebral infarction.

\section{Methods}

DW-MRI is a well-established clinical tool that provides unique information on the viability of the brain tissue. This sequence produces MRI based quantitative maps of the microscopic, natural displacements of water molecules that occur in the brain tissues as a part of the physical diffusion process. Water molecules are used as probes that reveal microscopic details about the architecture of both healthy and unhealthy brain tissues[19, 20]. The rate of water diffu- sion in the brain tissue is a direct function of its physiological state, and impacted by brain pathologies, such as cerebral infarction. There is an increased restriction to water mobility observed in the area of infarction within the brain tissue, and this produces a bright imaging appearance in the area of cerebral infarction when compared to the other healthy areas of the brain tissue[21]. The observed signal intensity peaks in 3 - 4 days and may be persistently high as long as 14 days after the onset of infarction. Signal intensity further decreases with time and reverts to normal by the end of the second week[18]. Accordingly detection of the areas of the brain with high signal intensity variations on the DW images subsequent to infarction, may allow for the evaluation of the affected area and further quantification of the observed signal intensity may prove to be useful in the early diagnosis and characterization of the different stages of cerebral infarction.

\subsection{Diffusion Imaging Protocol and Clinical Data}

All the subjects underwent clinical MR imaging with $1.5 \mathrm{~T}$ symphony maestro class MR scanning system from Siemens. DW imaging was performed by using a multisection, single-shot, spin-echo, echo-planar pulse sequence with following parameters: $\mathrm{TR}=3200 \mathrm{~ms}, \mathrm{TE}=94 \mathrm{~ms}$, acquisition matrix $=128 \times 128, \mathrm{FOV}=230 \mathrm{~mm} \times 230 \mathrm{~mm}$, and diffusion gradient value of $\mathrm{b}=1000 \mathrm{~s} / \mathrm{m} 2$ along 19 axial sections, 5 $\mathrm{mm}$ thick section and intersection gap of $1.5 \mathrm{~mm}$.

For the purpose of our study 40 cases ( 31 male, 09 female, ages 29 - 93 years with an average of age of 66 years) of cerebral infarction were diagnosed and verified with radiology data. MRI and DW imaging examinations were performed on eight subjects within 24 hours of symptom onset; eight subjects between days 1 and 4; fifteen subjects between days 5 and 9; six subjects between days 10 and 14; and three subjects after day 15 . For all the subjects with cerebral infarction considered, we confirmed the appearance of infarction by ruling out the possibility of bright intensity due to $\mathrm{T} 2$ shine through effects. This was done by verifying the appearance of hyperintensities on DW images and concomitant-reduced ADCs relative to the contralateral normal brain in their initial MRI studies. The ethics approval was obtained from the committee of clinical research at the RAGAVS diagnostic and Research center, Bangalore, India, and Vikram Hospital, Bangalore, India.

\subsection{Light Intensity High Frequency Power}

DW-MRI makes it to visualize and measure the altered rates of water diffusion, by producing a bright imaging appearance in the area of cerebral infarction. Consequently on examining the spatial intensity variation distribution on the DW images for the subjects with cerebral infarction, it is observed that the light signal intensity is not uniformly distributed over the entire DW image. There are abrupt jumps in the light signal intensity in the area of infarction in contrast to the other healthy areas of the brain where the light signal intensity distribution is almost uniform. Exploring the power 
spectrum of the DW intensity image for the subject with cerebral infarction would thereby result in a higher value for the higher spatial frequency power component out of the total power spectrum, in the area of infarction, when compared to the higher spatial frequency power component on the contralateral normal (healthy) hemisphere (mirror image) for the same subject. Consequently the light intensity HFP value in the area of infarction is much elevated when compared to the light intensity HFP value on the contralateral normal hemisphere for the same subject. The relative light intensity HFP values[RHFP = (maximum HFP value on infarct side - corresponding HFP value on contralateral side) / corresponding HFP value on contralateral side] are quantified across different subjects diagnosed with cerebral infarction and are employed in differentiating infarct tissues from healthy tissues and further used in the categorization of the different stages of cerebral infarction.

\subsection{Image Analysis}

The DW images obtained in DICOM format are converted to bmp format with intensities scaled to fit the conventional range of (0-255). This is done to reduce the complexity in the image manipulation algorithms and to achieve speed up in processing the images. The DW image obtained from the subject in the axial plane is divided into six anatomically significant areas as shown in Figure 1.

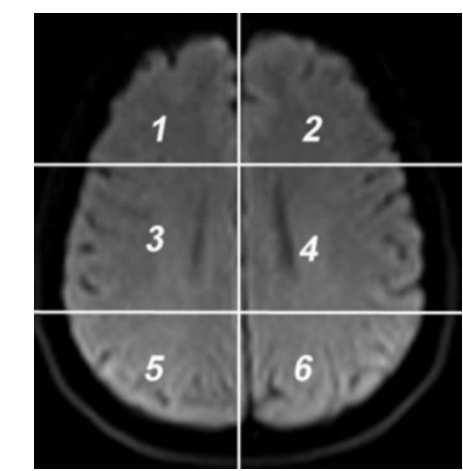

Figure 1. Axial DW image showing areas

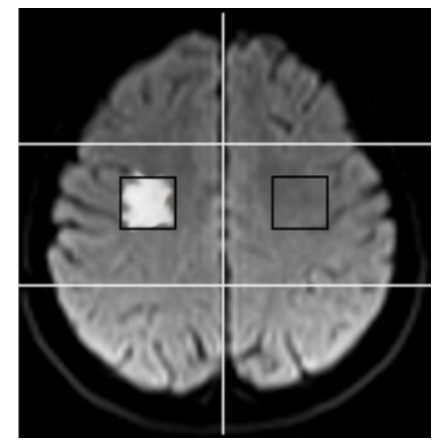

Figure 2. Axial DW image showing ROI on the infarct side (area 3, axial section 10) and contralateral normal hemisphere

For each subject with cerebral infarction, we obtain a set of DW images in the axial plane taken at different axial sections (from 1 to 19). The subject with cerebral infarction presents abrupt changes in the light signal intensities in one (or more) of the six areas in the affected axial sections. The axial sections that indicate such abrupt changes in the light signal intensities are selected for image analysis. For each axial section selected from the infarct subject, the following procedure is employed to obtain a decisive light intensity HFP value for that subject. Firstly a Region of Interest (ROI), $I(x, y)$, corresponding to the region of cerebral infarction is identified in the particular area of the axial section. An equivalent (mirror) ROI of the same size is selected on the contralateral normal hemisphere from the same subject for comparison[22] as shown in Figure 2 (infarction observed in area 3 at axial section 10 ).

The ROIs on each side are further divided into smaller sub-regions, $f(x, y)$, corresponding to an image size represented by $(\mathrm{M} \times \mathrm{N})$ pixels. The Fourier spectrum, $F(u, v)$, of an image, $f(x, y)$, corresponding to a sub-region of the ROI is evaluated using (1).

$$
F(u, v)=\frac{1}{M N} \sum_{x=0}^{M-1} \sum_{y=0}^{N-1} f(x, y) e^{\left[-j 2 \pi\left(\frac{u x}{M}+\frac{v y}{N}\right)\right]}
$$

for, $u=0,1,2 \ldots . M-1$ and $v=0,1,2 \ldots . N-1$

The spatial frequencies and their distribution for these sub-regions are analysed by performing the two-dimensional Discrete Fourier Transform (DFT) using MATLAB version 7.7. The spatial frequencies ( $u$ and $v$ ) are denoted by cycles per pixel, since the image size (distance) for the analysis is given in terms of pixels. Using the periodicity property of DFT[23], the Fourier spectrum is shifted to the center of the frequency plane. The DC component, $F(0,0)$ is deleted, since it gives only the average value of the image intensity. The power spectrum is obtained by squaring the magnitudes of the Fourier spectrum light intensity variations[23, 24] of the DW image and the total power, $T P$, in the image is obtained using (2). Since, for the sub-regions of the brain, the values of $\mathrm{M}$ and $\mathrm{N}$ are different (depend on the size of the particular sub-region), the cut-off frequency, $\mathrm{D}_{0}$ (in cycles per pixel), which separates the lower and higher spatial frequency components (as shown in Figure 3), is defined by (3).

$$
\begin{gathered}
T P=\left\{\sum_{u=-\frac{M}{2}}^{\frac{M}{2}} \sum_{v=-\frac{N}{2}}^{\frac{N}{2}}|F(u, v)|^{2}\right\}-|F(0,0)|^{2} \\
D_{0}=\left\{\begin{array}{cc}
\frac{M}{4} & \text { if } N \geq M \\
\frac{N}{4} & \text { if } N<M
\end{array}\right.
\end{gathered}
$$

Where $D(u, v)$ is the distance from the point $(u, v)$ to the origin of the frequency plane, defined by (4).

$$
D(u, v)=\sqrt{u^{2}+v^{2}}
$$

The Low Frequency Power (LFP) and HFP are calculated using (5) and (6) respectively. 


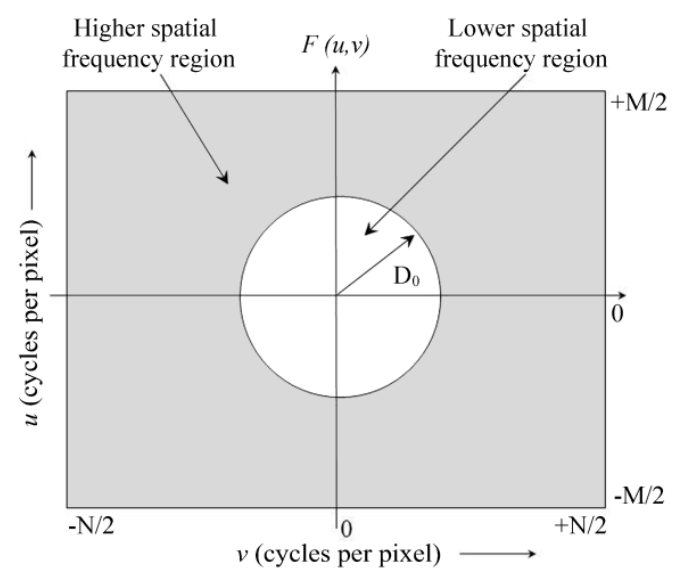

Figure 3. The Fourier spectrum, $F(u, v)$, of an image showing the higher and lower spatial frequency regions

$$
\begin{gathered}
L F P=\left\{\sum_{D(u, v)=0}^{D_{0}}|F(u, v)|^{2}\right\}-|F(0,0)|^{2} \\
H F P=T P-L F P
\end{gathered}
$$

For each sub-region on the infarct side and the corresponding sub-region on the contralateral normal side, the magnitude and the location of the light intensity HFP value is evaluated. The maximum light intensity HFP value over the entire ROI for the axial section on the infarct side is obtained. The corresponding light intensity HFP value from the contralateral normal hemisphere is noted for comparison. The magnitude of the maximum light intensity HFP value so obtained from the infarct subject indicates whether there is any sudden change in the signal intensity level in any of the sub-regions of the ROI for that axial section, and if there is any, then we can readily establish the sub-region, and also the exact location $((\mathrm{x}, \mathrm{y})$ co-ordinates) within the sub-region. The process is repeated for all the axial sections selected for image analysis from the infarct subject. After obtaining the maximum light intensity HFP values for all the axial sections, the average value of maximum light intensity HFP across the axial sections is chosen as the decisive light intensity HFP value for that infarct subject. The equivalent average value of the light intensity HFP from the contralateral normal hemisphere is evaluated. The method is repeated for all the subjects with cerebral infarction considered under study, and finally the light intensity RHFP value for each infarct subject is evaluated.

Statistical study is carried out by using the SPSS 17.0 software package. The analysis is performed at $99 \%$ confidence level $(p<0.01)$ using the significant difference of means method[25]. The average variations of the HFP values for the subjects with cerebral infarction are compared to their corresponding HFP values from the contralateral normal hemisphere. Further comparison is carried out between the mean RHFP values at different time stage of infarction (as shown in Table 1). Additionally the correlation between the RHFP values and the different time stages of cerebral in- farction is evaluated.

\section{Results and Discussions}

The DW image for a subject showing infarction in area 3 and at axial section 8 is shown in Figure 4.

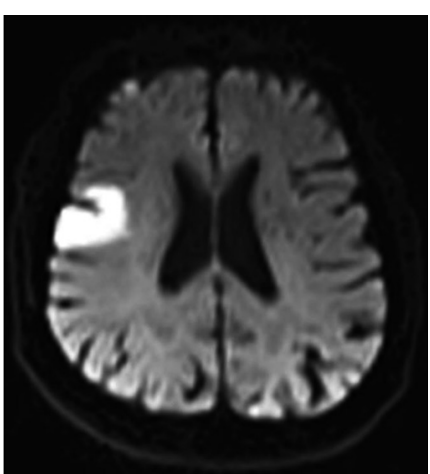

Figure 4. Axial DW image showing infarction in area 3

To carry out image analysis an ROI is carefully placed on the DW image in area 3, which corresponds to infarction and an equivalent ROI in the contralateral normal hemisphere. Both the ROIs are divided uniformly into smaller sub-regions. For each sub-region of the subject with infarction, the light intensity HFP value is evaluated and at the same time the corresponding HFP value from the contralateral normal hemisphere is noted down. Later the maximum light intensity HFP value is obtained across all the sub-regions for that axial section. The plot of the spatial variation of intensity distribution for a sub-region of the ROI in area 3, at axial section 8 resulting in maximum HFP value for the infarct subject is shown in Figure 5. The corresponding power spectrum is shown in Figure 6.

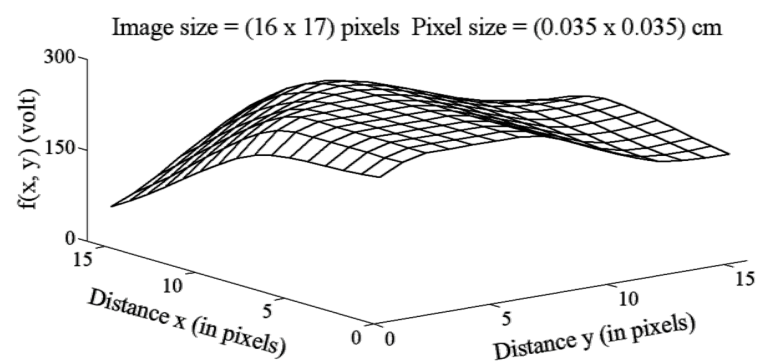

Figure 5. Image intensity distribution for the subject with infarct in area 3

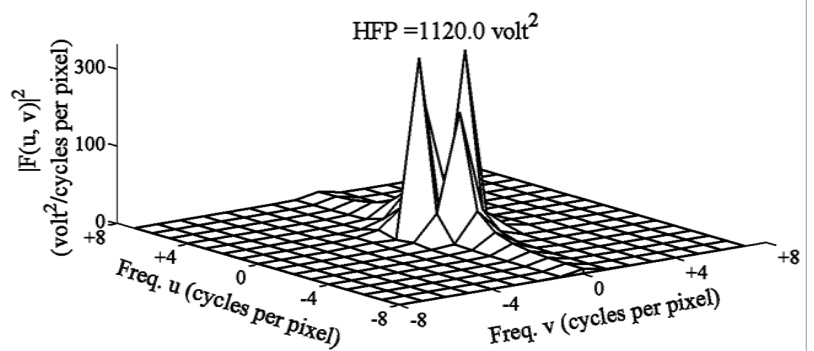

Figure 6. Power spectrum for the subject with infarct in area 3

It is observed from Figure 5 that the spatial intensity variation distribution for the subject with infarction has abrupt jumps and this leads to higher value of the higher 
spatial frequency component out of the total power spectrum. Similarly Figure 7 and Figure 8, respectively, show the plots of the spatial variation of intensity distribution and the power spectrum for the corresponding sub-region on the contralateral normal hemisphere for the same subject. The spatial intensity variation distribution is almost uniform in the contralateral hemisphere as shown in Figure 7, and this leads to a considerable smaller value of the higher spatial frequency component out of the total power spectrum as shown in Figure 8.

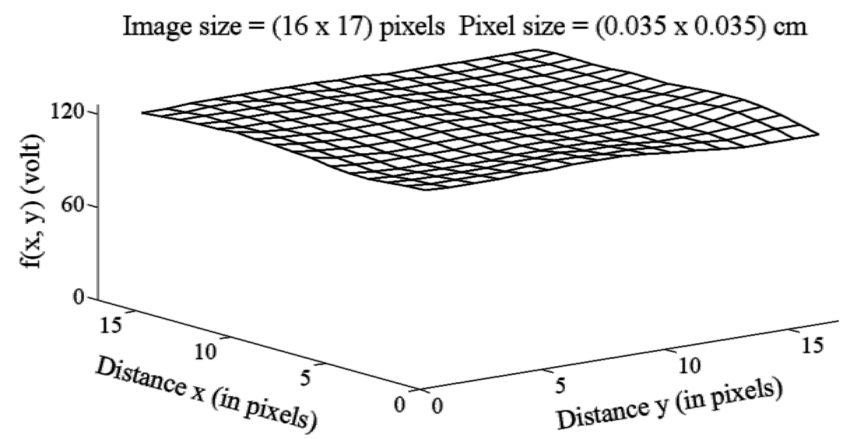

Figure 7. Image intensity distribution on the contralateral hemisphere

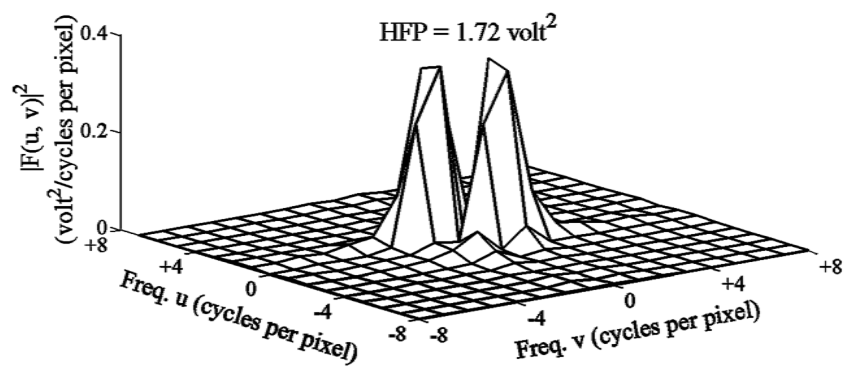

Figure 8. Power spectrum on the contralateral hemisphere

Consequently maximum HFP value for the subject with infarction $\left(1120.0\right.$ volt $\left.^{2}\right)$ is much elevated as shown in Figure 6 compared to that of the contralateral normal hemisphere $\left(1.72\right.$ volt $\left.^{2}\right)$ as shown in Figure 8. The maximum value of the HFP parameter evaluated indicates the maximum change in the light intensity variation across the selected ROI at the axial section 8 and also indicates its exact location, $(11,1)$, in the (x, y) co-ordinates (Figure 5).

The above procedure is carried out across all the axial sections selected from the infarct subject and eventually the average value of the maximum HFP across all the axial sections is taken as the decisive HFP parameter. The plot of the variation in the average light intensity HFP value in the brain infarcted area and the corresponding average light intensity HFP value from the contralateral normal hemisphere, for all the 40 subjects considered in the study is shown in Figure 9.

The difference in the average light intensity HFP values for the subjects with infarction compared to their contralateral normal hemispheres, were highly significant $(\mathrm{p}<0.01)$ in the areas of the brain, where there was a high incidence of cerebral infarction. The results suggest that the average light intensity HFP values obtained from the DW images for the subjects with cerebral infarction can be used to clearly differentiate them from the normal subjects.

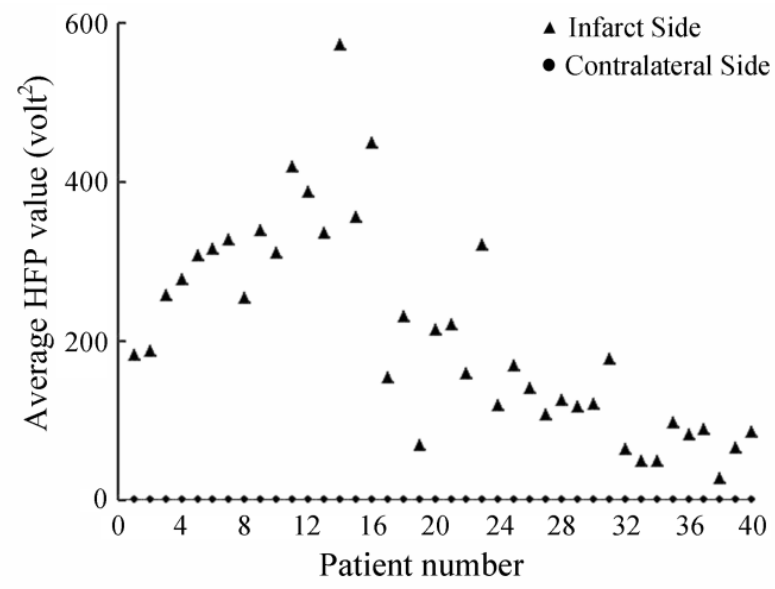

Figure 9. Variation in the average HFP values for the infarct subjects with the corresponding average HFP values from the contralateral hemisphere

The range of the average HFP values in the area of cerebral infarction and the contralateral normal hemisphere as well as the mean RHFP values for the subjects in each time phase of infarction compared to their contralateral normal hemisphere is shown in Table 1. The variation in the mean RHFP values for each set of subjects within the different time stages of cerebral infarction (last column of Table 1) is plotted in Figure 10.

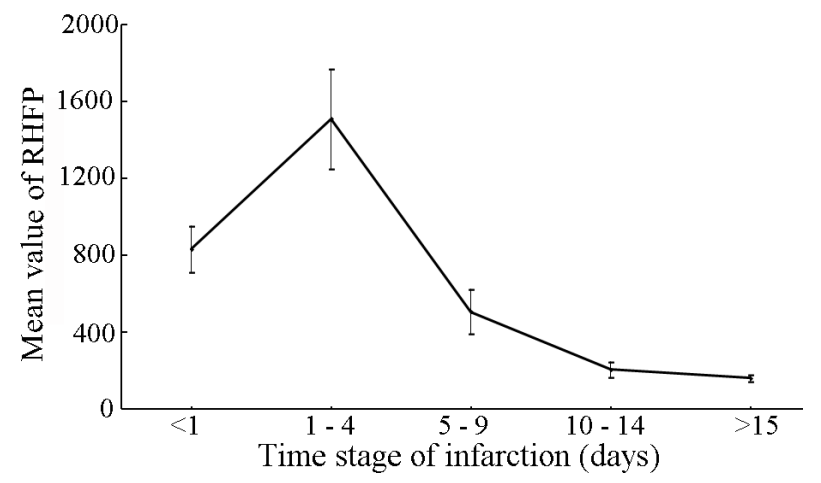

Figure 10. Variation in the mean RHFP values with the time stage of cerebral infarction

It is observed that there is an initial increase in the RHFP values and the maximum value for RHFP is obtained for the set of subjects whose examination is between 1 to 4 days. Further there is a decrease in the RHFP values and they reach their minimum for examinations after 15 days. This is in accordance with the pathophysiological changes following brain damage post cerebral infarction. There is also a significant difference $(p<0.01)$ observed between the mean RHFP values at different time stages of infarction as listed in Table 1. The plot of the variation in the RHFP values for the infarct subjects (40 cases) with the time stage of infarction is shown in Figure 11. The line given by the equation $y=-3.26$ $x+1185.19$, represents the linear regression that fit the data. 
Table 1. Average HFP and Mean RHFP* values in different time stage of cerebral infarction

\begin{tabular}{|c|c|c|c|c|}
\hline \multirow{2}{*}{ Time (days) } & \multirow{2}{*}{ Number of Cases } & \multicolumn{2}{|c|}{ Average HFP Value (volt2) } & \multirow{2}{*}{ Mean RHFP Value (x $\pm \mathrm{s}$ ) } \\
\cline { 3 - 4 } & & Infarct Side & Contralateral Side & \\
\hline$<1$ & 8 & $181.63 \sim 327.18$ & $0.2031 \sim 0.3836$ & $834.40 \pm 119.69$ \\
\hline $1-4$ & 8 & $311.07 \sim 573.48$ & $0.2029 \sim 0.3386$ & $1510.88 \pm 258.27$ \\
\hline $5-9$ & 15 & $67.87 \sim 321.18$ & $0.1373 \sim 0.4107$ & $508.37 \pm 116.23$ \\
\hline $10-14$ & 6 & $47.64 \sim 96.93$ & $0.2870 \sim 0.4113$ & $207.74 \pm 40.14$ \\
\hline$>15$ & 3 & $26.38 \sim 85.29$ & $0.1712 \sim 0.4679$ & $162.47 \pm 16.28$ \\
\hline
\end{tabular}

* RHFP = (maximum HFP value on infarct side - corresponding HFP value on contralateral side) / (corresponding HFP value on contralateral side)

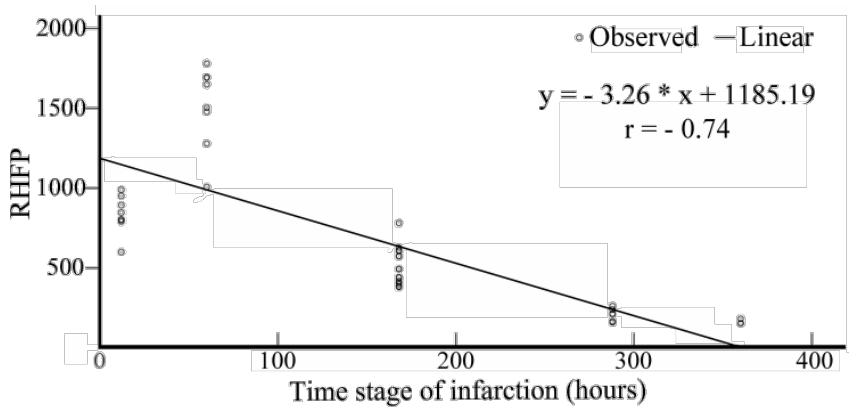

Figure 11. Variation in the RHFP values with the time stage of cerebral infarction

A negative correlation $(\mathrm{r}=-0.74)$ is observed between the RHFP values and different time stages of cerebral infarction.

The evolution of the RHFP values observed subsequent to infarction is suggestive that they can be supportive in understanding the developmental stages of infarction and can positively be helpful in predicting infarct growth. The results obtained in our study largely coincide with the findings of the previously published work using ADC values to monitor development of infarction[1,17]. However unlike the methods using ADC values, where only a particular axial section from the subject is considered for investigation, we have included all the axial sections that show hyperintensity due to infarction in order to arrive at the final RHFP value. Further for each axial section selected, the entire infarcted area is taken into consideration in contrast to the ADC methods where-in the ADC values are usually evaluated at the center of the infarcted area[10, 12].

\section{Conclusions}

In summary, the present study provides evidence that the light intensity HFP values on the DW images in the region of cerebral infarction can be used to markedly distinguish the infarct subjects quantitatively from the normal subjects. The relative increase in the light intensity HFP values on the DW images for the subjects with cerebral infarction were in the range of $(153.06$ - 1780.43) times compared to their corresponding HFP values on the contralateral normal hemisphere. The quantitative changes in the values of the light intensity HFP parameter on the DW images can be assessed and positively employed to provide useful information about the early changes taking place in the brain tissue and can be helpful in determining the stage of cerebral infarction. Therefore the adoption of the proposed quantitative method in the clinical diagnosis and treatment of cerebral infarction could be helpful and instructive.

\section{ACKNOWLEDGEMENTS}

The authors would like to thank Dr. Sudhir Pai (Sr. Consultant, Neurosurgeon, Vikram Hospital, Bangalore, India) for assistance in data collection and Dr. Deepak Y. S. (D.N.B. Resident, RAGAVS Diagnostic and Research Center, Bangalore, India) for his valuable comments and suggestions.

\section{REFERENCES}

[1] Jian-Min Shen, Xian-Wu Xia, Wu-Gen Kang, Jian-Jun Yuan, Liang Sheng, "The Use of MRI Apparent Diffusion Coefficient (ADC) in Monitoring the Development of Brain Infarction”, BMC Medical Imaging, vol. 11, no. 2, 2011.

[2] S. Warach, D. Chien, W. Li, M. Ronthal, R. R. Edelman, "Fast Magnetic Resonance Diffusion-Weighted Imaging of Acute Human Stroke", Neurology, vol. 42, no. 9, pp. 1717-1723, 1992.

[3] S. Warach, J. Gaa, B. Siewert, P. Wielopolski, R. R. Edelman, "Acute Human Stroke Studied by Whole Brain Echoplanar Diffusion-Weighted Magnetic Resonance Imaging", Annals of Neurology, vol. 37, no. 2, pp. 231-241, 1995.

[4] S. Chabert, P. Scifo, "Diffusion Signal in Magnetic Resonance Imaging: Origin and Interpretation in Neurosciences", Biological Research, vol. 40, no. 4, pp. 385-400, 2007.

[5] Le Bihan D., "Looking into the Functional Architecture of the Brain with Diffusion MRI", Nature Reviews Neuroscience, vol. 4, no. 6, pp. 469-480, 2003.

[6] Y. Yoneda , K. Tokui, T. Hanihara, T. Kitagaki, M. Tabuchi, E. Mori, "Diffusion-Weighted Magnetic Resonance Imaging: 
Detection of Ischemic Injury 39 Minutes After Onset in a Stroke Patient", Annals of Neurology, vol. 45, no. 6, pp. 794-797, 1999.

[7] W. T. Yuh, M. R. Crain, D. J. Loes, G. M. Greene, T. J. Ryals, Y. Sato, "MR Imaging of Cerebral Ischemia: Findings in the First 24 Hours", American Journal of Neuroradiology, vol. 12, pp. 621-629, 1991.

[8] H. L. Lutsep, G. W. Albers, A. DeCrespigny, G. N. Kamat, M. P. Marks, M. E. Moseley, "Clinical Utility of Diffusion-Weighted Magnetic Resonance Imaging in the Assessment of Ischemic Stroke", Annals of Neurology, vol. 41, no. 5, pp. 574-580, 1997.

[9] T. W. Stadnik, P. Demaerel, R. R. Luypaert, C. Chaskis, K. L. Van Rompaey, A. Michotte, M. J. Osteaux, "Imaging Tutorial: Differential Diagnosis of Bright Lesions on Diffusion-Weighted MR Images," Radiographic, vol. 23, no. 3, 2003.

[10] G. Schlaug, B. Siewert, A. Benfield, R. R. Edelman, S. Warach, "Time Course of the Apparent Diffusion Coefficient (ADC) Abnormalities in Human Stroke", Neurology, vol. 49, no. 1, pp. 113-119, 1997.

[11] M. G. Lansberg, V. N. Thijs, M. W. O'Brien, J. O. Ali, A. J. de Crespigny, D. C. Tong, M. E. Moseley, G. W. Albers, "Evolution of Apparent Diffusion Coefficient, Diffusion Weighted, and T2-Weighted Signal Intensity of Acute Stroke", American Journal of Neuroradiology, vol. 22, no. 4, pp. 637-644, 2001

[12] D. Gyu Na, V. N. Thijs, G. W. Albers, M. E. Moseley, M. P. Marks, "Diffusion-Weighted MR Imaging in Acute Ischemia: Value of Apparent Diffusion Coefficient and Signal Intensity Thresholds in Predicting Tissue at Risk and Final Infarct Size", American Journal of Neuroradiology, vol. 25, no. 8, pp. 1331-1336, 2004.

[13] W. A. Copen, L. H. Schwamm, R. G. Gonzalez, O. Wu, C. B. Harmath, P. W. Schaefer, W. J. Koroshetz, A. G. Sorensen, "Ischemic Stroke: Effects of Etiology and Patient Age on Time Course of the Core Apparent Diffusion Coefficient", Radiology, vol. 221, pp. 27-34, 2001.of Infarct Signal Intensity on Diffusion-Weighted Images", American Journal of Neuroradiology, vol. 24, pp. 680-687, 2003.

[14] P. M. Desmond, A. C. Lovell, A. A. Rawlinson, M. W. Parsons, P. A. Barber, Q. Yang, T. Li, D. G. Darby, R. P. Gerraty, S. M. Davis, B. M. Tress, "The Value of Apparent Diffusion Coefficient Maps in Early Cerebral Ischemia", American Journal of Neuroradiology, vol. 22, pp. 1260-1267, 2001.

[15] L. H. Schwamm, W. J. Koroshetz, A. G. Sorensen, B. Wang,
W. A. Copen, R. Budzik, G. Rordorf, F. S. Buonanno, P. W. Schaefer, R. G. Gonzalez, "Time Course of Lesion Development in Patients with Acute Stroke: Serial Diffusion and Hemodynamic-Weighted Magnetic Resonance Imaging", Stroke, vol. 29, no. 11, pp. 2268-2276, 1998.

[16] A. K. Srivastava, G. Mehrotra, S. K. Bhargava, S. Agarwal, R. P. Tripathi, "Studies on the Time Course of Apparent Diffusion Coefficient and Signal Intensities on T2- and Diffusion-Weighted MR Imaging in Acute Cerebral Ischemic Stroke", Journal of Medical Physics, vol. 33, no. 4, pp. 162-170, 2008.

[17] J. D. Eastwood, S. T. Engelter, J. F. MacFall, D. M. Delong, J. M. Provenzale, "Quantitative Assessment of the Time Course of Infarct Signal Intensity on Diffusion-Weighted Images", American Journal of Neuroradiology, vol. 24, pp. 680-687, 2003.

[18] J. H. Burdette, P. E. Ricci, N. Petitti, A. D. Elster, “Cerebral Infarction: Time Course of Signal Intensity Changes on Diffusion-Weighted MR Images", American Journal of Roentgenology, vol. 171, no. 3, pp. 791-795, 1998.

[19] L. C. Maas, P. Mukherjee, "Diffusion MRI: Overview and Clinical Applications in Neuroradiology", Applied Radiology, vol. 34, no. 11, pp. 44-60, 2005.

[20] R. Rajeshkannan, S. Moorthy, K. P. Sreekumar, R. Rupa, N. K. Prabhu, "Clinical Applications of Diffusion Weighted MR Imaging: A Review", Indian Journal of Radiology and Imaging, vol. 16, no. 4, pp. 705-710, 2006.

[21] S. Holtas, B. Geijer, L. G. Stromblad, P. Maly-Sundgren, I. M. Burtscher, "A Ring-Enhancing Metastasis with Central High Signal on Diffusion-Weighted Imaging and Low Apparent Diffusion Coefficients", Neuroradiology, vol. 42, no. 11, pp. 824-827, 2000.

[22] D. A. Kulkarni, Bhagyashree S. M., G. R. Udupi, "Texture Analysis of Mammographic Images", International Journal of Computer Applications, vol. 5, pp. 12-17, 2010.

[23] Gonzalez R. C., Wintz P., Image transforms in 'Digital Image Processing', $2^{\text {nd }}$ edition, Addison-Wesley, U.S.A, 1987.

[24] K. G. Prabhu, K. M. Patil, S. Srinivasan, "Diabetic Feet at Risk: A New Method of Analysis of Walking Foot Pressure Images at Different Levels of Neuropathy for Early Detection of Plantar Ulcers", Medical and Biological Engineering and Computing, vol. 39, pp. 288-293, 2001.

[25] S. C. Gupta, V. K. Kapoor, Fundamentals of Mathematical Statistics, $11^{\text {th }}$ edition, Sultan Chand and Sons, New Delhi, India, 1982. 